\title{
Seroquel Induced Liver Injury
}

Muhammad Tahir ${ }^{1^{*}}$ and Paula G Burkard ${ }^{2}$

${ }^{1}$ Department of Internal Medicine, Sisters of Charity Hospital, Buffalo, NY, USA

${ }^{2}$ Department of Gastroenterology, University at Buffalo, NY, USA

Corresponding author: Muhammad Tahir, Department of Internal Medicine, Sisters of Charity hospital, 2157 Main St, Buffalo, NY, USA, Tel: (716) 862-1000; E-mail: muhammadtahir090@gmail.com

Received date: September 06, 2017; Accepted date: September 26, 2017; Published date: September 28, 2017

Copyright: (C) 2017 Tahir M, et al. This is an open-access article distributed under the terms of the Creative Commons Attribution License, which permits unrestricted use, distribution, and reproduction in any medium, provided the original author and source are credited.

\begin{abstract}
The drug induced liver injury by antipsychotics is a well reported subject in the medical literature. The hepatotoxic effects have been reported by atypical antipsychotic drugs like clozapine, risperidone and olanzapine [1]. The Quetiapine induced hepatocellular damage is relatively a rare incident. It is an atypical antipsychotic agent proven to be effective for the management of negative and positive symptoms of the psychosis [2]. It is a dibenzothiazepine derivative which works by mediating through a combined antagonism of dopamine type 2 (D2) and serotonin type 2 (5-HT2) receptors. Although considering its wide therapeutic range up to $750 \mathrm{mg} / \mathrm{day}$, it has a low side effect profile [3]. The common side effects reported are asymptomatic liver enzymes elevation, pancytopenia and thrombotic thrombocytopenic purpura [4]. The rare side effects reported are Neuroleptic malignant syndromes [5] and cardiac abnormalities in some of the patients. In our case report, we will describe the incident of acute liver injury caused by quetiapine in our patient, which if not identified and treated earlier can cause significant morbidity and mortality.
\end{abstract}

\section{Keywords DILI; Trazodone; Hepatotoxicity; Liver failure}

\section{Case Report}

A 28 year old African American male with past medical history of bipolar disorder \& antisocial personality disorder presented to the ED with reported overdose of his anti-psych medication pills associated with inflicting superficial lacerations to his left forearm. Although patient reasoned it just for sleep-aid, he had significant history of multiple suicidal attempts with the same pattern of drug overdose and self-inflicting injuries. Patient was started on Seroquel 2 weeks ago after which he had on \& off complaint of weakness. In ED, the patient only complained of nausea after the incident. Otherwise, denied fever, rigors/chills, vomiting or hematemesis, change in the color of stool, abdominal pain, chest pain, and shortness of breath or any other drug overdose. Patient had a 10 pack year history of smoking tobacco with on and off marijuana use, but denied alcohol intake.

The result of general physical examination revealed a completely stable hemodynamic status. The abdominal examination was benign with no pain, tenderness or any stigmata of chronic liver disease. The cardiovascular and respiratory exam was unremarkable. The mental state examination MSE was normal with a score of $>24$ indicating a normal cognition.

The laboratory workup on admission showed a normal blood cell count and normal renal function values including electrolytes However, it revealed a significant increase in the liver enzymes (ALT/ SGPT: 1824Units/L; AST/SGOT: 921Units/L; Alkaline phosphatase: 120Units/L). The total bilirubin level was $0.9 \mathrm{mg} / \mathrm{dL}$ with an INR of 1.1. The electrocardiogram monitoring was done to assess QTc as there is a risk for prolonged QTc and torsades by the reported quetiapine overdose which was reported in presentation. The patient blood alcohol level was found to be within the normal limits; also the autoimmune workup and viral serology were found negative. The blood and urine-10 toxicology was found unremarkable for any of the drugs. The result of screening test for Hemochromatosis and Wilson disease was also negative.

The USG abdomen showed a normal homogeneous echo-texture with no masses or dilation of the intrahepatic bile ducts. The gallbladder was normal with no gallstones, pericholecystic fluid, or gallbladder wall thickening. The size of liver and spleen was documented WNL. The CT scan endorsed the findings of USG.

Following this workup and history findings, the patient was admitted in the hospital with a probable diagnosis of Quetiapine induced hepatocellular injury. The initial treatment was supportive and Quetiapine was discontinued; rather temazepam was started by psychiatry department for insomnia and stabilization of psychiatric illness. Continuous monitoring of complete metabolic panel was done. The liver biopsy was deferred as per guidelines in literature i.e. if testing for alternative causes of liver injury is negative and the patient has been exposed to a drug known to be associated with hepatic injury, we typically do not proceed with a liver biopsy.

Over the in-patient hospital course, the biochemical framework improved significantly as shown in the graphical presentation in Figure 1. After 1 week of inpatient stay, patient was discharged in stable condition with advice against taking quetiapine. The blood workup, during the follow up visit after 4 weeks, was found normal.

Patient has been doing fine currently on outpatient follow up. 


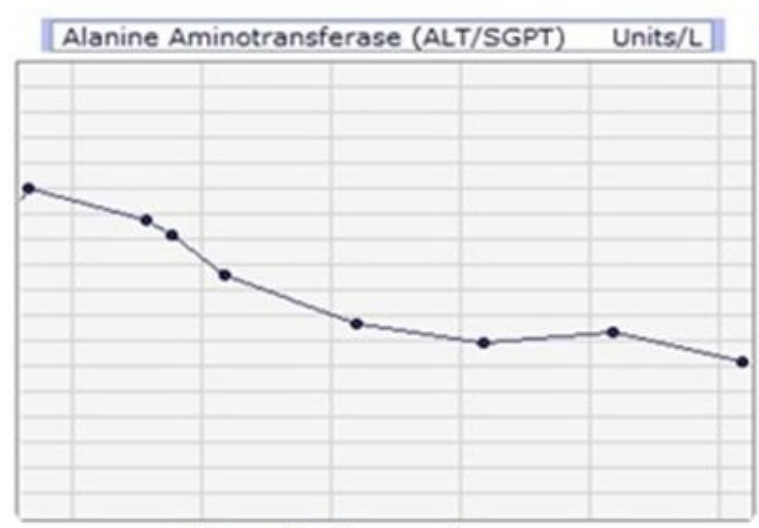

ALT units/L trend

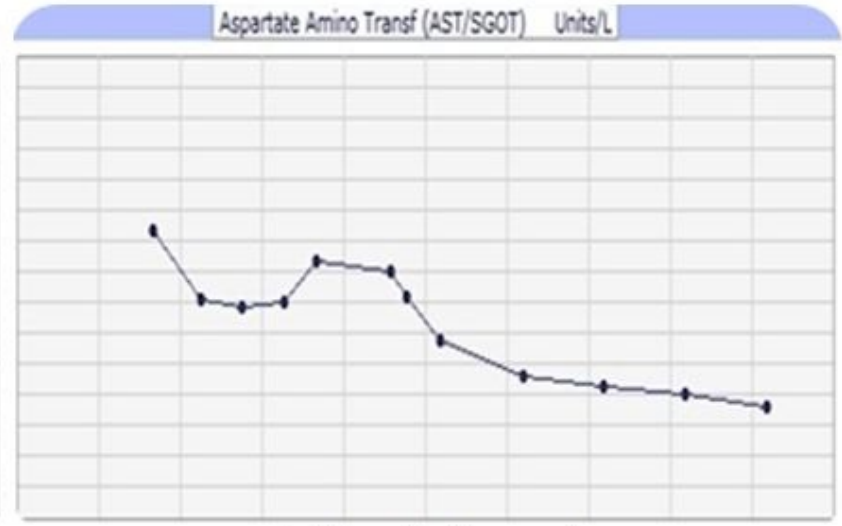

AST units/L trend

Figure 1: ALT units/Ltrend and AST units/Ltrend shown.

\section{Discussion}

It has been estimated that more than 600 drugs and chemicals have been associated with significant liver injury. The drug induced liver injury is one of the most common causes of fulminant hepatic failure. Quetiapine was initially reported benign regarding hepatotoxicity except for a mild asymptomatic elevation of liver enzymes prior to the first published report of quetiapine-induced FHF by El Hajj et al. in 2004 [6]. The most of the drugs are metabolized in the liver by the CYP3A subfamily of the enzymes. The toxicity of a drug can be potentially enhanced either by reduced conversion to its non-toxic metabolites or by increase in its conversion to toxic ones. This is possibly the reason, some drugs are not harmful within therapeutic range but are reported otherwise. Also, genetic variation can predispose some of the patients to particular drug adverse reactions. The hepatotoxic effects can either by direct cellular injury or idiosyncratic reaction (immune-mediated) [7]. Although the mechanism and the biochemical reactions that lead to the cellular injury are still unclear but theory revolved around the production of free radicals and reactive oxygen species (ROS). There is no reference reported between the amount of the drug and the time when patient starts taking the drug to the severity of the reaction [8].

The gold standard for the diagnosis is to re-challenge the patient with the offending substance, however it is not advised. The mainstay of the treatment remains withdrawal of the drug and conservative measures. The recovery should be expected after stopping the drug but prognosis varies in patients i.e. worse in those who presents with jaundice. Sometimes injury continues even after withholding the drug therapy and leads to fibrosis or cirrhosis of the liver. AHF by idiosyncratic mechanism has a very bad prognosis with a mortality rate around $80 \%$ without transplantation [9].

Review of the literature described cases in which patients were mostly taking some concurrent medications potentially believed to cause or aggravate the liver dysfunction i.e. trazodone, risperidone, lithium etc. [6]. However, our patient was not taking any other medication besides Seroquel which makes us conclude that he had drug-induced hepatocellular damage, given that it improved after stopping the drug.

\section{Learning point}

Considering the world wide use of the quetiapine, physicians should always be aware of the serious hepatic consequences associated with the drug. Also, care should be taken to reconcile the patient home medications. We should always consider the medications being taken by the patient as the potential cause. This is why medication reconciliation is very vital part of the clinical care.

\section{References}

1. Wright TM, Vandenberg AM (2007) Risperidone- and quetiapineinduced cholestasis. Ann Pharmacother 41: 1518-1523.

2. Lacy C, Armstrong L, Goldman M, Lance L (2001) Quetiapine. Drug Information Handbook 9 edition Lexi-comp Inc, Hudson, $\mathrm{OH}$ pp: 1046-1047.

3. Mullen J, Jibson MD, Sweitzer D (2001) A comparison of the relative safety, efficacy, and tolerability of quetiapine and risperidone in outpatients with schizophrenia and other psychotic disorders: the quetiapine experience with safety and tolerability (QUEST) study. Clin Ther 23: $1839-1854$.

4. Iraqi A (2003) A case report of pancytopenia with quetiapine use. Am J Geriatr Psychiatry 11: 694.

5. Bourgeois JA, Babine S, Meyerovich M, Doyle J (2002) A case of neuroleptic malignant syndrome with quetiapine. J Neuropsychiatry Clin Neurosci 14: 87.

6. El Hajj I, Sharara AI, Rockey DC (2004) Subfulminant liver failure associated with quetiapine. Eur J Gastroenterol Hepatol 16: 1415-1418.

7. Kaplowitz N (2004) Drug-induced liver injury. Clin Infect Dis 38: S44.

8. Shpaner A, Li W, Ankoma-Sey V, Botero RC (2008) Drug-induced liver injury: hepatotoxicity of quetiapine revisited. Eur J Gastroenterol Hepatol 20: 1106-1109.

9. Lee WM (2003) Drug-induced hepatotoxicity. N Engl J Med 349: 474-485. 